International Journal of Pure and Applied Mathematics

Volume 106 No. 2 2016, 639-647

ISSN: 1311-8080 (printed version); ISSN: 1314-3395 (on-line version)

url: http://www.ijpam.eu

doi: 10.12732/ijpam.v106i2.26

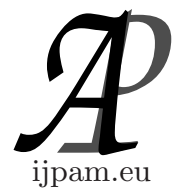

\title{
OSCILLATORY AND NONOSCILLATORY BEHAVIOUR OF SOLUTIONS OF GENERALIZED MIXED DIFFERENCE EQUATIONS
}

\author{
M. Maria Susai Manuel ${ }^{1}$, G. Dominic Babu ${ }^{2}$, \\ D.S. Dilip ${ }^{3}$, G. Britto Antony Xavier ${ }^{4}$ \\ ${ }^{1}$ Department of Mathematics, R.M.D. Engineering College, \\ Kavaraipettai - 601 206, Tamil Nadu, S. INDIA \\ ${ }^{2}$ Department of Mathematics \\ Annai Velankanni College \\ Tholaiyavattam, Kanyakumari District, INDIA \\ ${ }^{3}$ Department of Mathematics \\ St. John's College, Anchal, Kollam Dt., INDIA \\ ${ }^{4}$ Department of Mathematics \\ Sacred Heart College \\ Tirupattur, 635 601, Vellore District,Tamil Nadu, INDIA
}

Abstract: In this paper, the authors discuss the oscillatory and nonoscillatory behaviour of solutions of some generalized mixed difference equations of the form

$$
\begin{aligned}
& \Delta_{\ell}^{2}\left(\Delta_{\alpha(\ell)} u(k)\right)+\delta p(k) u(k)=0, k \in[a, \infty), \\
& \Delta_{\ell}^{3}\left(\Delta_{\alpha(\ell)} u(k)\right)+\delta p(k) u(k)=0, k \in[a, \infty),
\end{aligned}
$$

where $\delta= \pm 1$ and the function $p$ is real with $p(k) \geq c$ and $\alpha, \ell$ are positive real.

AMS Subject Classification: 39A12

Key Words: generalized difference operator, mixed difference equation, oscillation and nonoscillation

Received: November 16, 2015

Published: February 18, 2016

${ }^{\S}$ Correspondence author
(C) 2016 Academic Publications, Ltd. url: www.acadpubl.eu 


\section{Introduction}

The basic theory of difference equations is based on the operator $\Delta$ defined as $\Delta u(k)=u(k+1)-u(k), k \in \mathbb{N}=\{0,1,2,3, \cdots\}$. Eventhough many authors (see [1], [19]-[23]) have suggested the definition of $\Delta$ as

$$
\Delta u(k)=u(k+\ell)-u(k), k \in \mathbb{R}, \ell \in \mathbb{R}-\{0\},
$$

no significant progress took place on this line. But recently, E. Thandapani, M.M.S. Manuel, G.B.A.Xavier [7] considered the definition of $\Delta$ as given in (3) and developed the theory of difference equations in a different direction. For convenience, the operator $\Delta$ defined by (3) is labelled as $\Delta_{\ell}$ and by defining its inverse $\Delta_{\ell}^{-1}$, many interesting results and applications in number theory were obtained. By extending the study related to the sequences of complex numbers and $\ell$ to be real, some new qualitative properties of the solutions like rotatory, expanding, shrinking, spiral and weblike were obtained for difference equation involving $\Delta_{\ell}$. The results obtained using $\Delta_{\ell}$ are found in (see [7]-[14],[17],[18]).

Jerzy Popenda and B. Szmanda (see [5],[6]) defined $\Delta$ as

$$
\Delta_{\alpha} u(k)=u(k+1)-\alpha u(k)
$$

and based on this definition they studied the qualitative properties of a particular difference equation and no one else has handled this operator.

In [15] the authors extended the definition of $\Delta_{\alpha}$ to $\Delta_{\alpha(\ell)}$ defined on $u(k)$ as $\Delta_{\alpha(\ell)} v(k)=v(k+\ell)-\alpha v(k)$, where $\alpha \neq 0, \ell>0$ are fixed and $k \in[0, \infty)$ is variable. By defining the inverse $\Delta_{\alpha(\ell)}^{-1}$, several interesting results on number theory were obtained (see [12],[14],[15],[16]).

An equation involving both $\Delta$ and $\Delta_{\alpha}$ is called mixed difference equation. Oscillatory behaviour of solutions of certain types of mixed difference equations have been dicussed in $[3,4,21,22]$. An equation involving $\Delta_{\ell}$ and $\Delta_{\alpha(\ell)}$ is called as generalized mixed difference equation.

B. Smith and W.E. Taylor (see [21]) investigated the oscillatory behavior of solutions of certain mixed difference equations.

In this paper the theory is extended from $\Delta$ to $\Delta_{\ell}$ and $\Delta_{\alpha}$ to $\Delta_{\alpha(\ell)}$ for all real $k \in[a, \infty)$ and we discuss the oscillatory and nonoscillatory behavior of solutions of the generalized mixed difference equations (1) and (2).

Throughout this paper, we make use the following assumptions:

(i) $\mathbb{N}_{\ell}(j)=\{j, j+\ell, j+2 \ell, \ldots\}$.

(ii) $\lceil x\rceil$ and $[x]$ denote upper integer and integer part of $x$ respectively. 
(iii) $j=k-k_{i}-\left[\frac{k-k_{i}}{\ell}\right] \ell, k_{i} \in[0, \infty)$.

\section{Preliminaries}

In this section, we present some preliminaries of generalized difference operator and its inverse which will be useful for future discussion.

Definition 2.1. [7] Let $u(k), k \in[0, \infty)$, be a real or complex valued function and $\ell>0$ be fixed. Then, the inverse of $\Delta_{\ell}$ denoted by $\Delta_{\ell}^{-1}$ is defined as follows;

$$
\text { If } \Delta_{\ell} v(k)=u(k), \text { then } v(k)=\Delta_{\ell}^{-1} u(k)+c_{j}
$$

where $c_{j}$ is a constant for all $k \in \mathbb{N}_{\ell}(j), j=k-\left[\frac{k}{\ell}\right] \ell$.

In general $\Delta_{\ell}^{-n} u(k)=\Delta_{\ell}^{-1}\left(\Delta_{\ell}^{-(n-1)} u(k)\right)$ for the integers $n \geq 2$.

Definition 2.2. [11] The inverse of the Generalized $\alpha$-difference operator, denoted by $\Delta_{\alpha(\ell)}^{-1}$, on $u(k)$ is defined as follows. If $\Delta_{\alpha(\ell)} v(k)=u(k)$, then

$$
\Delta_{\alpha(\ell)}^{-1} u(k)=v(k)-\alpha^{\left[\frac{k}{\ell}\right]} v(j)
$$

where $k \in \mathbb{N}_{\ell}(j), j=k-\left[\frac{k}{\ell}\right] \ell$.

Lemma 2.3. $[7]$ (Finite Summation formula) If the real valued function $u(k)$ is defined for all $k \in[0, \infty)$, then

$$
\left.\Delta_{\ell}^{-1} u(k)\right|_{j} ^{k}=\sum_{r=1}^{\left[\frac{k}{\ell}\right]} u(k-r \ell)+c_{j}
$$

where $c_{j}$ is a constant for all $k \in \mathbb{N}_{\ell}(j), j=k-\left[\frac{k}{\ell}\right] \ell$.

Definition 2.4. [11] The solution $u(k)$ of a generalized difference equation is called oscillatory if for any $k_{1} \in[a, \infty)$ there exists a $k_{2} \in \mathbb{N}_{\ell}\left(k_{1}\right)$ such that $u\left(k_{2}\right) u\left(k_{2}+\ell\right) \leq 0$. The difference equation itself is called oscillatory if all its solutions are oscillatory. If the solution $u(k)$ is not oscillatory, then it is said to be nonoscillatory (i.e. $u(k) u(k+\ell)>0$ for all $\left.k \in\left[k_{1}, \infty\right)\right)$. 


\section{Main Results}

The higher order $\left(\mathrm{n}^{\text {th }}\right.$ order) generalized $\alpha_{i}$ - difference equation of the form $\Delta_{\alpha_{1}\left(\ell_{1}\right)}\left(\Delta_{\alpha_{2}\left(\ell_{2}\right)}\left(\cdots \Delta_{\alpha_{n}\left(\ell_{n}\right)}(v(k)) \cdots\right)\right)=u(k), k \in[0, \infty), \ell_{i}>0 \alpha_{i} \neq 0$ becomes generalized mixed difference equation if $\alpha_{i}=1$ for some $i$ and $n \geq 2$. In this section we study the asymptotic behavior of the non-oscillatory solutions of the generalized mixed difference equation (1) and (2).

Theorem 3.1. Suppose $u(k)$ is a nonoscillatory solution of equation (1) if

$$
\operatorname{sgn} u(k)=\operatorname{sgn} \Delta_{\ell}^{2} u(k) \neq \operatorname{sgn} \Delta_{\ell} u(k)=\operatorname{sgn} \Delta_{\ell}^{3} u(k)
$$

and

$$
\lim _{n \rightarrow \infty} u(k)=0
$$

Proof. A nonoscillatory solution of (1) may not exist if $0<\alpha<1$, but if it does exist, we show that it must satisfy (8) and (9). As the negative solution of equation (1) is also a solution of the same equation, it suffices to prove that a positive solution of (1) satisfies (8). Let $u(k)>0$ be a non-oscillatory solution of (1) for $\delta=1$.

Setting $r(k)=\Delta_{\alpha(\ell)} u(k)=u(k+\ell)-\alpha u(k)$, we get

$$
\Delta_{\ell}^{2} r(k)=-p(k) u(k)<0,
$$

and so $\Delta_{\ell} r(k)$ is (eventually) strictly decreasing. From (10) it follows that if $\Delta_{\ell} r(k)$ is eventually negative we must have $r(k) \rightarrow-\infty$. However this is contradictory, since $r(k)=u(k+\ell)-\alpha u(k)=\Delta_{\ell} u(k)+(1-\alpha) u(k) \rightarrow-\infty$ implies $\Delta_{\ell} u(k)$, forces $u(k)$ to be eventually negative. Hence we must have

$$
\Delta_{\ell} r(k)>0
$$

for all large $k$. Indeed we will show that $\lim _{k \rightarrow \infty} u(k)=0$.

Writing (1) as $\Delta_{\ell}^{2}\left(\Delta_{\alpha(\ell)} u(k)\right)=-p(k) u(k)$, and by Lemma 2.3 , when $k_{0}$ is chosen large enough so that $\Delta_{\ell} r(k)>0$ for all $k \geq k_{0}$, we get

$$
\Delta_{\ell} r(k)-\Delta_{\ell} r\left(k_{0}\right)=-\sum_{r=0}^{\left[\frac{k-\ell}{\ell}\right]} p\left(k_{0}+r \ell\right) u\left(k_{0}+r \ell\right) .
$$

The liminf condition on $p(k)$ yields

$$
0<c \sum_{r=0}^{\left[\frac{k-\ell}{\ell}\right]} u\left(k_{0}+r \ell\right) \leq \sum_{r=0}^{\left[\frac{k-\ell}{\ell}\right]} p\left(k_{0}+r \ell\right) u\left(k_{0}+r \ell\right)<\Delta_{\ell} r(k) .
$$


Letting $k \rightarrow \infty$, we see that $\sum_{r=0}^{\infty} u\left(k_{0}+r \ell\right)<\infty$ and therefore $\lim _{k \rightarrow \infty} u(k)=0$. Since $u(k) \rightarrow 0$ as $k \rightarrow \infty$ it follows that $r(k) \rightarrow 0$ as $k \rightarrow \infty$. From (11) we get $r(k)$ is increasing and hence $r(k)<0$ eventually. It then follows from the inequality $r(k)=\Delta_{\ell} u(k)+(1-\alpha) u(k)<0$ that $\Delta_{\ell} u(k)<0$ and from (11) we obtain the relation $\Delta_{\ell} r(k)=\Delta_{\ell}^{2} u(k)+(1-\alpha) \Delta_{\ell} u(k)>0$ and $\Delta_{\ell}^{2} u(k)>0$. From (10) we get $\Delta_{\ell}^{2} r(k)=\Delta_{\ell}^{3} u(k)+(1-\alpha) \Delta_{\ell}^{2} u(k)<0, \Delta_{\ell}^{3} u(k)<0$ and the proof is complete.

Example 3.2. The solution of the third order generalized mixed difference equation

$$
\begin{aligned}
\Delta_{\ell}^{2}\left(\Delta_{\alpha(\ell)} u(k)\right) & \\
+\frac{(k+2 \ell)_{\ell}^{(3)}-(2+\alpha)(k+3 \ell)(k+\ell)_{\ell}^{(2)}+(1+2 \alpha)(k+3 \ell)_{\ell}^{(2)} k}{(k+3 \ell)_{\ell}^{(3)}} & \times u(k+3 \ell)_{\ell}^{(3)} \\
& \times 0,
\end{aligned}
$$

satisfies all the conditions of Theorem 3.1 and $\lim _{n \rightarrow \infty} u(k)=0$. Infact $u(k)=\frac{1}{k}$ is one such solution.

Theorem 3.3. If $u(k)$ is a nonoscillatory solution of (1) for $\delta=-1$ with $\alpha>1$ then for all $k$ sufficiently large

$$
\operatorname{sgn} u(k)=\operatorname{sgn} \Delta_{\ell} u(k)=\operatorname{sgn} \Delta_{\ell}^{2} u(k),
$$

and

$$
\lim _{k \rightarrow \infty}|u(k)|=\lim _{k \rightarrow \infty}\left|\Delta_{\ell} u(k)\right|=\lim _{k \rightarrow \infty}\left|\Delta_{\ell}^{2} u(k)\right|=\infty .
$$

Proof. Assume that $u(k)>0$ for all $k$ sufficiently large.

Taking $r(k)=\Delta_{\alpha(\ell)} u(k)=u(k+\ell)-\alpha u(k)$, we get

$$
\Delta_{\ell}^{2} r(k)=p(k) u(k)>0
$$

and $\Delta_{\ell} r(k)$ is increasing. If $\Delta_{\ell} r(k)$ is eventually positive, then as $k \rightarrow \infty, r(k) \rightarrow$ $\infty$ and since $r(k)=\Delta_{\ell} u(k)+(1-\alpha) u(k)$ and $\alpha>1$ it follows that $\Delta_{\ell} u(k) \rightarrow \infty$, which in turn implies $u(k) \rightarrow \infty$. To see $\Delta_{\ell}^{2} u(k) \rightarrow \infty$, note that $u(k) \rightarrow \infty$ implies $\Delta_{\ell}^{2} r(k) \rightarrow \infty$ and $\Delta_{\ell} r(k) \rightarrow \infty$ because of (14). Hence the result follows from $\Delta_{\ell} r(k)=\Delta_{\ell}^{2} u(k)+(1-\alpha) \Delta_{\ell} u(k)$. Now, if $\Delta_{\ell} r(k)$ is eventually negative and increasing, then $\Delta_{\ell} r(k)$ has a limit as $k \rightarrow \infty$. However $\Delta_{\ell} r(k)$ having a 
limit implies that $\sum_{k=0}^{\infty} u\left(k_{0}+r \ell\right)<\infty$ and this implies $u(k) \rightarrow 0$. But $u(k) \rightarrow 0$ implies $r(k) \rightarrow 0$ and since $r(k)$ is decreasing to zero we get $r(k)>0$. But the relation $r(k)=\Delta_{\ell} u(k)+(1-\alpha) u(k)>0$ implies $\Delta_{\ell} u(k)>0$, a contradiction since $u(k)>0$ and $\Delta_{\ell} u(k)>0$ is inconsistent with $u(k) \rightarrow 0$. Hence (13) cannot have a nonoscillatory solution with $\Delta_{\ell} r(k) \Delta_{\ell}^{2} r(k)<0$ for all $k$ sufficiently large.

Example 3.4. The Theorem 3.3 holds for the generalized mixed difference equation

$$
\Delta_{\ell}^{2}\left(\Delta_{\alpha(\ell)} u(k)\right)+\frac{2 \ell^{2}(1-\alpha)}{k^{2}} u(k)=0 .
$$

Infact $u(k)=k^{2}$ is one solution.

Theorem 3.5. Consider the equation (2) for $\delta=-1$ and $\alpha \geq 1$. If $u(k)$ is a nonoscillatory solution, then for all $k$ sufficiently large either

$$
\operatorname{sgn} u(k)=\operatorname{sgn} \Delta_{\ell} u(k)=\operatorname{sgn} \Delta_{\ell}^{2} u(k)=\operatorname{sgn} \Delta_{\ell}^{3} u(k)
$$

or

$$
\operatorname{sgn} u(k)=\operatorname{sgn} \Delta_{\ell}\left(\Delta_{\alpha(\ell)} u(k)\right) \neq \operatorname{sgn} \Delta_{\alpha(\ell)} u(k)=\operatorname{sgn} \Delta_{\ell}^{2}\left(\Delta_{\alpha(\ell)} u(k)\right) .
$$

Proof. We prove the case for $\alpha>1$. Assume $u(k)$ is eventually positive. Taking $r(k)=\Delta_{\alpha(\ell)} u(k)=u(k+\ell)-\alpha u(k)$, we get

$$
\Delta_{\ell}^{3} r(k)=p(k) u(k)>0 .
$$

Clearly $\Delta_{\ell}^{2} r(k)$ is increasing. In case $\Delta_{\ell}^{2} r(k)$ is eventually positive, we will have $\lim _{k \rightarrow \infty} \Delta_{\ell} r(k)=\lim _{k \rightarrow \infty} r(k)=\infty$ and since $r(k)<u(k+\ell)$ it follows that $u(k) \rightarrow \infty$. Since $p(k)>c$ for large $k$, we have

$$
\lim _{k \rightarrow \infty} \Delta_{\ell}^{3} r(k)=\lim _{k \rightarrow \infty} \Delta_{\ell}^{2} r(k)=\infty .
$$

Since $r(k)=\Delta_{\ell} u(k)+(1-\alpha) u(k) \rightarrow \infty$ and $\alpha>1$ it follows that $\Delta_{\ell} u(k) \rightarrow \infty$. Examining $\Delta_{\ell} r(k)=\Delta_{\ell}^{2} u(k)+(1-\alpha) \Delta_{\ell} u(k)$ we see that $\Delta_{\ell}^{2} u(k) \rightarrow \infty$ as $k \rightarrow \infty$. Continuing in this manner we see that (15) holds eventually.

Next, we consider the case where $\Delta_{\ell}^{3} r(k)>0$ and $\Delta_{\ell}^{2} r(k)<0$. Then, existence of $\lim _{k \rightarrow \infty} \Delta_{\ell}^{2} r(k)$ and Lemma 2.3 yield 


$$
\begin{aligned}
-\Delta^{2} r\left(k_{1}\right)>\Delta^{2} r(m)-\Delta^{2} r\left(k_{1}\right)=\sum_{t=0}^{\frac{k-\ell-k_{1}-j}{\ell}} p\left(k_{1}+j+t \ell\right) u\left(k_{1}+j+t \ell\right) \\
\geq c \sum_{t=0}^{\frac{k-\ell-k_{1}-j}{\ell}} u\left(k_{1}+j+t \ell\right) .
\end{aligned}
$$

Letting $m \rightarrow \infty$, it then follows that $\sum_{t=0}^{\infty} u\left(k_{1}+j+t \ell\right)<\infty$ and hence $\lim _{k \rightarrow \infty} u(k)=0$ which implies $r(k) \rightarrow 0$. Thus, if $\Delta_{\ell}^{3} r(k)>0$ and $\Delta_{\ell}^{2} r(k)<0$ then we have

$$
\Delta_{\ell}^{3} r(k)>0, \Delta_{\ell}^{2} r(k)<0, \Delta_{\ell} r(k)>0,
$$

because $\Delta_{\ell}^{2} r(k)<0$ and $\Delta_{\ell} r(k)<0$ is inconsistent with $r(k) \rightarrow 0$, it then follows that either (i) $r(k)>0$ or (ii) $r(k)<0$ eventually. We will show that (i) is impossible. If (i) holds then since $r(k)=\Delta_{\ell} u(k)+(1-\alpha) u(k)>0$, it follows that $\Delta_{\ell} u(k)>0$, in fact we have that $\Delta_{\ell} u(k)>c+(\alpha-1) u(k)>c$ for some positive constant $c$ and so, $u(k) \rightarrow \infty$ as $k \rightarrow \infty$. But this implies $\Delta_{\ell}^{3} r(k) \rightarrow \infty$, so we must have $\Delta_{\ell}^{2} r(k)>0$ eventually, contradicting (18). So (i) cannot hold, resulting (ii) holding eventually.

Example 3.6. The Theorem 3.5 holds for the generalized mixed difference equation

$$
\Delta_{\ell}^{3}\left(\Delta_{\alpha(\ell)} u(k)\right)+\frac{6 \ell^{3}(1-\alpha)}{k^{3}} u(k)=0 .
$$

Infact $u(k)=k^{3}$ is one such solution.

Theorem 3.7. Every nontrivial bounded solution of (2) for $\delta=1$, where $\alpha>1$, is oscillatory.

Proof. Suppose $u(k)>0$ is bounded nonoscillatory for large $k$.

Letting $r(k)=\Delta_{\alpha(\ell)} u(k)=u(k+\ell)-\alpha u(k)$, we see that $r(k) \geq-\alpha u(k)$ and then

$$
\Delta_{\ell}^{3} r(k)=-p(k) u(k)<0 .
$$

Obviouly $\Delta^{2} r(k)$ is decreasing and if $\Delta^{2} r(k)$ is eventually negative, we see that $r(k) \rightarrow \infty$. This clearly contradicts the boundedness of $u(k)$. Thus, we consider the case where $\Delta^{2} r(k)>0$. In this case, $\lim _{k \rightarrow \infty} \Delta^{2} r(k)=t \geq 0$. Using the fact $p(k)$ is bounded away from zero for large $k$, it follows that $\Delta_{\ell} r(k)<0$ and $r(k)>0$ for large $k$. Furthermore $\lim _{k \rightarrow \infty} u(k)=0$, since $\Delta_{\ell}^{2} r(k) \rightarrow t$ implies 
$\sum_{r=0}^{\infty} u(r \ell)<\infty$. Since $\alpha>1$ and $r(k)=\Delta_{\ell} u(k)+(1-\alpha) u(k)>0, \Delta_{\ell} u(k)>0$ for all $k$ sufficiently large. But this is a contradiction, since $u(k) \Delta_{\ell} u(k)>0$ is congruent with $u(k) \rightarrow 0$.

Example 3.8. The Fourth generalized mixed difference equation

$$
\Delta_{\ell}^{3}\left(\Delta_{\alpha(\ell)} u(k)\right)+\frac{\left(1+\alpha^{2}\right)(1+\alpha)^{3}}{\alpha^{4}} u(k)=0,
$$

satisfies the conditions of Theorem 3.7 and hence the solution is oscillatory. Infact $u(k)=\frac{1}{(-\alpha)^{\left\lceil\frac{k}{\ell}\right\rceil}}$ is one such solution.

\section{Acknowledgments}

Research Supported by National Board for Higher Mathematics, Department of Atomic Energy, Government of India, Mumbai.

\section{References}

[1] R.P. Agarwal, Difference Equations and Inequalities, Marcel Dekker, New York, 2000.

[2] Aleksandra Sternal and Blazej Szmanda, Asymptotic and Oscillatory Behaviour of Certain Difference Equations, Le Matematiche, LI(1996), Fasc.I, 77-86.

[3] S.R. Grace, Oscillation of Certain Neutral Difference Equations of Mixed Type, Journal of Math Analysis Applications., 1998, 224: 241-254.

[4] S.R. Grace and S. Donatha Oscillation of Higher Order Neutral Difference Equations of Mixed Type, Dynam Syst Appl., 2003, 12: 521-532.

[5] Jerzy Popenda and Blazej Szmanda, On the Oscillation of Solutions of Certain Difference Equations, Demonstratio Mathematica, XVII(1), (1984), 153 - 164.

[6] Jerzy Popenda, Oscillation and Nonoscillation Theorems for Second-Order Difference Equations, Journal of Mathematical Analysis and Applications, 123(1), (1987), 34 - 38.

[7] M. Maria Susai Manuel, G. Britto Antony Xavier and E. Thandapani, Theory of Generalized Difference Operator and Its Applications, Far East Journal of Mathematical Sciences, 20(2), (2006), 163-171.

[8] M. Maria Susai Manuel, G. Britto Antony Xavier and E. Thandapani, Qualitative Properties of Solutions of Certain Class of Difference Equations, Far East Journal of Mathematical Sciences, 23(3) (2006), 295-304.

[9] M. Maria Susai Manuel and G. Britto Antony Xavier, Recessive, Dominant and Spiral Behaviours of Solutions of Certain Class of Generalized Difference Equations, International Journal of Differential Equations and Applications, 10(4) (2007), 423-433. 
[10] M. Maria Susai Manuel, Adem Kilicman, G. Britto Antony Xavier, R. Pugalarasu and D.S. Dilip, $\ell_{2(\ell)}$ and $c_{0(\ell)}$ solutions of a second order generalized difference equation, Advances in Difference Equation, Submitted.

[11] M. Maria Susai Manuel, G. Britto Antony Xavier, D.S. Dilip and G. Dominic Babu, Oscillation, Nonoscillation and Growth of Solutions of Generalized Second Order Nonlinear $\alpha$-Difference Equations, Global Journal of Mathematical Sciences: Theory and Practical, 4(1), 2012, $211-225$.

[12] M. Maria Susai Manuel, G. Britto Antony Xavier, D.S. Dilip and G. Dominic Babu, Oscillation and Nonoscillation for Certain Class of First and Second Order Generalized $\alpha$-Difference Equations, International Journal of Pure And Applied Mathematics, 78(3), 2012, $451-468$.

[13] M. Maria Susai Manuel, G. Britto Antony Xavier and D.S. Dilip, $\alpha$-Difference Operator And Its Application On Number Theory, J. of Mod. Meth. in Numer. Math., 3(2), (2012), $79-95$.

[14] M. Maria Susai Manuel, G. Britto Antony Xavier, D.S. Dilip and G. Dominic Babu , Solutions of Certain Type of Second Order Generalized $\alpha$-Difference Equation in $L_{p}$ Space, International Journal of Advances In Pure And Applied Mathematics, Accepted.

[15] M. Maria Susai Manuel, V. Chandrasekar and G. Britto Antony Xavier, Theory of Generalized $\alpha$-Difference Operator and its Applications in Number Theory, Advances in Differential Equations and Control Processes, 9(2) (2012), 141-155.

[16] M. Maria Susai Manuel, V. Chandrasekar and G. Britto Antony Xavier, Solutions and Applications of Certain Class of $\alpha$-Difference Equations, International Journal of Applied Mathematics, 24(6) (2011), 943-954.

[17] R. Pugalarasu, M. Maria Susai Manuel, V. Chandrasekar and G. Britto Antony Xavier, Theory of Generalized Difference operator of $n$-th kind and its applications in number theory (Part I), International Journal of Pure and Applied Mathematics, 64(1) (2010), 103-120.

[18] R. Pugalarasu, M. Maria Susai Manuel, V. Chandrasekar and G. Britto Antony Xavier, Theory of Generalized Difference operator of $n$-th kind and its applications in number theory (Part II), International Journal of Pure and Applied Mathematics, 64(1) (2010), 121-132.

[19] Ronald E.Mickens, Difference Equations, Van Nostrand Reinhold Company, New York, 1990.

[20] Saber N Elaydi, An Introduction To Difference Equations, Second Edition, Springer, 1999.

[21] B. Smith and W.E Taylor, Oscillation and Nonoscillation Theorems for some Mixed Difference Equations, International Journal of Math. and Math. Sci., 15(3), (1992), 537 - 542 .

[22] E. Thandapani and N. Kavitha, Oscillatory Behaviour of Solutions of Certain Third Order Mixed Neutral Difference Equations, Acta Mathematica Scientia, 33B(1), pp. 218-226, 2013.

[23] Walter G Kelley, Allan C Peterson, Difference Equations, An Introduction with Applications, Academic Press, inc 1991. 
